\title{
The gaussian distribution of inhomogeneous barrier heights in PtSi/p-Si Schottky diodes
}

\author{
Somayeh Gholami ${ }^{1 a)}$, Hassan Hajghassem ${ }^{2}$, and A. R. Erfanian ${ }^{3}$ \\ ${ }^{1}$ Dep. of Electrical Engineering, Islamic Azad University, Science and Research \\ Branch, Tehran, Iran \\ ${ }^{2}$ University of Shahid Beheshti, Tehran, Iran \\ ${ }^{3}$ K.N. Tossi University, Tehran, Iran \\ a)gholamirudi@ce.sharif.edu
}

Abstract: The current-voltage characteristics of PtSi/p-Si Schottky barrier diodes were investigated in the temperature range of 85 -136K and their barrier height $\left(\phi_{b}\right)$, ideality factor $(\mathrm{n})$ and series resistance $\left(R_{s}\right)$ were found to be strongly temperature-dependent. While n decreases, $\phi_{b}$ increases with increasing temperature and the conventional activation energy plot deviates from linearity. These discrepancies from ideal thermionic emission theory is attributed to Schottky barrier inhomogeneities and the Schottky barrier inhomogeneities are shown to obey a single Gaussian distribution with a mean value of $0.4548 \mathrm{eV}$ and a standard deviation of $0.0607 \mathrm{eV}$. The modified activation plot which takes into account the barrier height variations through the Gaussian distribution is acceptably linear and gives $\left(\overline{\phi_{b}}\right)$ and $A^{*}$ as $0.4546 \mathrm{eV}$ and $31.47 \mathrm{Acm}^{-2} \mathrm{~K}^{-2}$ respectively.

Keywords: Schottky barrier diodes, temperature dependence, barrier inhomogeneities, gaussian distribution

Classification: Optoelectronics, Lasers and quantum electronics, Ultrafast optics, Silicon photonics, Planar lightwave circuits

\section{References}

[1] J. R. Jimenez, X. Xiao, J. C. Sturm, and P. W. Pellegrini, "Tunable, longwavelength $\mathrm{PtSi} / \mathrm{SiGe} / \mathrm{Si}$ Schottky diode infrared detectors," Appl. Phys. Lett., vol. 67, pp. 506-508, 1995.

[2] J. P. Sullivan, R. T. Tung, M. R. Pinto, and W. R. Graham, "Electron transport of inhomogeneous Schottky barriers," J. Appl. Phys., vol. 70, pp. 7403-7424, 1991.

[3] S. Chand and J. Kumar, "On the existence of a distribution of barrier heights in Pd2Si/Si Schottky diodes," J. Appl. Phys., vol. 80, pp. 288294, 1996.

[4] S. Zeyrek, M. M. Bulbul, S. Altındal, and M. C. Baykul, "The double gaussian distribution of inhomogeneous barrier heights in $\mathrm{Al} / \mathrm{GaN} / \mathrm{p}-\mathrm{GaAs}$ Schottky diodes in wide temperature range," Brazilian J. Phys., vol. 38, p. $59,2008$. 
[5] I. Dökme, S. S. Altındal, and I. M. Afandiyeva, "The distribution of the barrier height in Al-TiW-Pd2Si/n-Si Schottky diodes from I-V-T measurements," Semicond. Sci. Technol., vol. 23, 2008.

[6] E. H. Rhoderick, Metal-Semiconductor Contacts, Clarendon, Oxford, 1988.

[7] D. Donoval, M. Barus, and M. Zdimal, "Analysis of I-V measurements on PtSi-Si Schottky structures in a wide temperature range," Solid-State Electron., vol. 34, pp. 1365-1373, 1991.

\section{Introduction}

Platinum Silicide films are extensively used in silicon devices to form Schottky barriers and ohmic contacts. Infrared detection is a special application of $\mathrm{PtSi} / \mathrm{p}-\mathrm{Si}$ Schottky barrier. PtSi/p-Si detector has received a lot of attention as the most promising infrared sensor for large focal plane array applications due to its ease of fabrication, uniformity, compatibility with today's IC technology and low cost [1].

The I-V-T analysis of Schottky diodes using pure thermionic emission theory usually reveals some abnormal behaviors such as the decrease of barrier height $(\mathrm{BH})$ and increase of ideality factor with decreasing temperature and the nonlinearity of the Richardson plot over a wide temperature range. Some authors have attributed the discrepancies to the barrier inhomogeneities [2]. Various types of distribution functions have been used to describe BH inhomogeneities, among them Gaussian distribution has been widely accepted and used. Chand and Kumar [3] have analyzed the I-V characteristics of $\mathrm{Pd}_{2} \mathrm{Si} / \mathrm{n}$ - Si Schottky diode and interpreted its abnormal behavior on the basis of the thermionic emission-diffusion theory and the assumption of a Gaussian distribution of barrier height. Similar works have been done on various Schottky diodes $[4,5]$.

In this work, first we investigated the temperature-dependency of electrical parameters of $\mathrm{PtSi} / \mathrm{p}-\mathrm{Si}$ Schottky diodes using their current-voltage (I-V) characteristics which was measured in the temperature range of 85136K. Experimental results showed that ideality factor, barrier height and series resistance were strong functions of temperature. Then the temperature dependence of the I-V characteristics of Schottky barrier diodes was explained on the basis of the thermionic emission theory with a Gaussian distribution of the inhomogeneous barrier heights.

\section{Experiment}

The diodes were fabricated on p-type (100) Si substrate with resistivity of $4-11 \Omega \mathrm{cm}$. The samples were chemically cleaned using the standard RCA cleaning procedure and rinsed in deionized water and dried. Before ohmic contacts formed on the p-type Si substrates, the samples were dipped in dilute $\mathrm{HF}: \mathrm{H}_{2} \mathrm{O}$ (1:10) for about $3 \mathrm{~min}$ to remove any native oxide layer on the surface, and finally the samples were rinsed with deionized water and 
dried with high purity nitrogen and inserted into the deposition chamber immediately after cleaning. To form ohmic contacts we thermally evaporated high purity $\mathrm{Al}$ with a thickness of $2060 \AA$ on the surface of the samples at the pressure of $10^{-5}$ mbar and then annealed the evaporated $\mathrm{Al}$ at $550^{\circ} \mathrm{C}$ for $5 \mathrm{~min}$ at the pressure of $7 \times 10^{-6}$ mbar. All the metal depositions were done through metal shadow masks. To fabricate PtSi layer, Pt film with a thickness of $80 \AA$ was evaporated with an electron gun system on the cleaned Si surfaces and samples were annealed in a vacuum of $7 \times 10^{-6}$ mbar at $470^{\circ} \mathrm{C}$ for $30 \mathrm{~min}$. To form the metal electrodes (rectifier and ohmic contacts), $\mathrm{Al}$ usually have been used. But $\mathrm{Al}$ has high diffusion ability and can lead to degradation of the contacts therefore in this work to prevent the problem of $\mathrm{Al}$ diffusion into $\mathrm{Si}$, we used $\mathrm{Ti}$ as a diffusion barrier between $\mathrm{PtSi}$ and $\mathrm{Al}$. $800 \AA$ of Ti was evaporated on PtSi layer at the pressure of $10^{-6}$ mbar and finally, Al films of $3000 \AA$ thickness were evaporated on Ti layer.

For the I-V measurement, the device was mounted on the cold work surface of a Dewar at liquid nitrogen temperature. An HP 4145B instrument was used to measure the I-V characteristics at the PtSi SBD.

\section{Results and discussion}

\subsection{Current-voltage characteristics of the PtSi/p-Si diode}

For a Schottky barrier diode with assumption that the current is due to thermionic emission, the relation between the applied voltage and current can be expressed as $[6]$

$$
I=I_{\circ} \exp \left(\frac{q V}{n k T}\right)\left[1-\exp \left(\frac{-q V}{k T}\right)\right]
$$

Eq. (1) for $V>3 k T / q$ can be written as:

$$
I=I_{\circ} \exp \left(\frac{q V}{n k T}\right)
$$

where $\mathrm{T}$ is the temperature in Kelvin, $\mathrm{q}$ the electronic charge, $\mathrm{k}$ is the Boltzmann constant and $I_{\circ}$ is the reverse saturation current which can be written as:

$$
I_{\circ}=A A^{*} T^{2} \exp \left(\frac{-q \phi_{b}}{k T}\right)
$$

where $A$ is the effective diode area, $A^{*}$ is the effective Richardson constant which is equal to $32 \mathrm{Acm}^{-2} \mathrm{~K}^{-2}$ for $\mathrm{p}$-Si and $\phi_{b}$ is the zero-bias barrier height. In Eq. (2), $\mathrm{n}$ is the ideality factor which is a parameter generally used to measure the deviation of practical diodes from ideal thermionic emission model.

Fig. 1 (a) shows the experimental I-V characteristics of our $\mathrm{PtSi} / \mathrm{p}-\mathrm{Si}$ diode at the temperature of $85 \mathrm{~K}$ in dark. It can be seen from Fig. 1 (a) that the current curve in forward bias quickly becomes dominated by series resistance and deviates from linearity. Series resistance $R_{s}$ which is an important parameter in the electrical characteristics of Schottky barrier diodes is the total resistance of bulk of the semiconductor, contact wires and ohmic contacts. For evaluating $\mathrm{n}, \phi_{b}$ and $R_{s}$, we modeled the real Schottky diode with 
an ideal diode in series with a resistance $R_{s}$. According to thermionic emission theory, $V_{D}$ which is the voltage across the metal-semiconductor interface in the Schottky diode can be written as

$$
V_{D}=\frac{n k T}{q} \operatorname{Ln}\left(\frac{I}{A A^{*} T^{2}}\right)+n \phi_{b}
$$

And $V$ which is the applied or experimentally measured voltage in the Schottky diode circuit considering the equation $V=V_{D}+R_{s} I$ can be expressed as

$$
V=\frac{n k T}{q} \operatorname{Ln}\left(\frac{I}{A A^{*} T^{2}}\right)+n \phi_{b}+I R_{s}
$$

Using Eq. (5) and the (I, V) experimental data, n, $\phi_{b}$ and $R_{s}$ can be determined.

$\mathrm{I}-\mathrm{V}$ characteristics of diode have been measured in the temperature range of $85-136 \mathrm{~K}$. The values of $\mathrm{n}, \phi_{b}$ and $R_{s}$ determined from the method described above are shown in Table I for each temperature. It is obvious that the values of $\mathrm{n}, \phi_{b}$ and $R_{s}$ are strong functions of temperature.

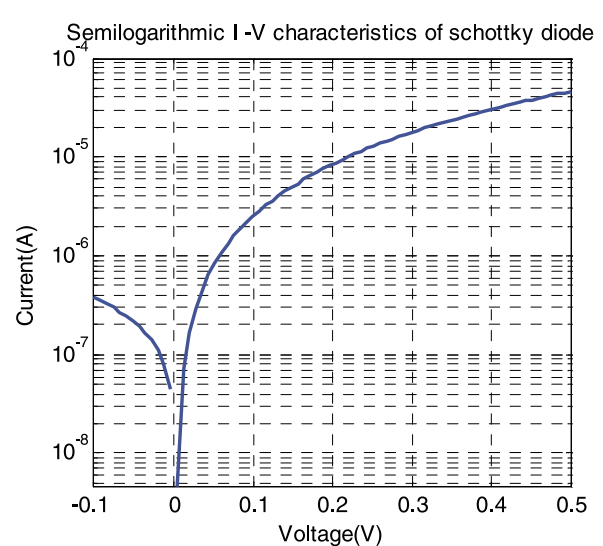

(a)

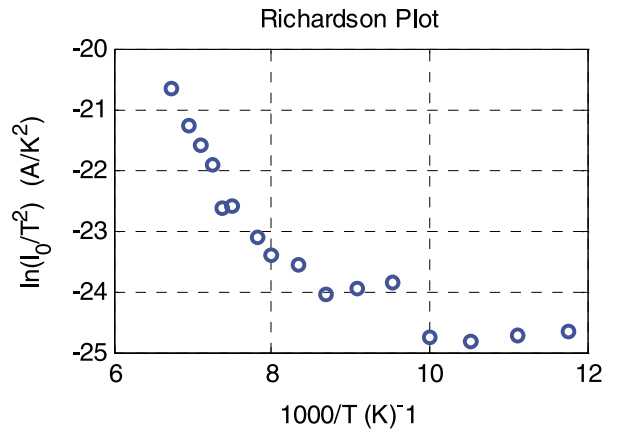

(b)

Fig. 1. (a) I-V characteristics of the PtSi/p-Si Schottky diode at the temperature of $85 \mathrm{~K}$ in dark and (b) Conventional activation energy plot of the $\mathrm{PtSi} / \mathrm{p}$ Si diode.

It can be seen from the experimental data given in Table I that series resistance $R_{s}$ strongly varies with temperature. The increase of series resistance with the fall of temperature is believed to result from lack of free charge carriers at low temperatures [7]. In our case the large values of $R_{s}$ at low temperatures can also be attributed to the nonideality of the ohmic contact.

It is also clear from Table I that with increasing temperature, barrier height is increased while ideality factor decreased.

Barrier height can also be evaluated by using the Arrhenius or Richardson plot of the saturation current. By taking the natural logarithm of Eq. (3), we will have

$$
\ln \left(\frac{I_{\circ}}{T^{2}}\right)=\ln \left(A A^{*}\right)-\frac{q \phi_{b}}{k T}
$$


Table I. Temperature dependent values of various experimental parameters obtained from I-V measurements of PtSi/p-Si Schottky diode.

\begin{tabular}{|c|c|c|c|}
\hline Temperature(Kelvin) & $\begin{array}{c}\text { Barrier height } \\
(\mathrm{eV})\end{array}$ & Ideality factor & $\begin{array}{c}\text { Series resistance } \\
(K \Omega)\end{array}$ \\
\hline 85 & 0.2044 & 2.9082 & 15.033 \\
\hline 90 & 0.2163 & 2.8613 & 14.629 \\
\hline 95 & 0.2296 & 2.7195 & 14.519 \\
\hline 100 & 0.2412 & 2.702 & 14.023 \\
\hline 105 & 0.2451 & 2.6743 & 10.426 \\
\hline 110 & 0.2576 & 2.6401 & 10.426 \\
\hline 115 & 0.2702 & 2.6278 & 10.426 \\
\hline 120 & 0.2772 & 2.5945 & 8.129 \\
\hline 125 & 0.2869 & 2.5626 & 7.455 \\
\hline 128 & 0.2907 & 2.5171 & 6.343 \\
\hline 133 & 0.2971 & 2.1903 & 5.115 \\
\hline 136 & 0.3019 & 2.158 & 5.115 \\
\hline
\end{tabular}

Plotting $\ln \left(I_{\circ} / T^{2}\right)$ versus $q / k T$ should give a straight line which its slope and intercept will give barrier height and Richardson constant respectively. Fig. 1 (b) Shows the conventional activation energy $\left(\ln \left(I_{\circ} / T^{2}\right)\right.$ versus $\left.1000 / T\right)$ plot, for the $\mathrm{PtSi} / \mathrm{p}$-Si diode based on the experimental data in Table I. It can be seen that the curve strongly deviates from linearity.

The discrepancies from classic thermionic emission theory observed in our PtSi/p-Si Schottky diode such as the increase of barrier height with increasing temperature and the nonlinearity of the activation energy plot can be remedied by considering spatial inhomogeneity of SBH. The spatial inhomogeneity is usually described by some distributed functions, among them Gaussian distribution is the most successful model to explain the nonideal behavior of Schottky diodes.

\subsection{Gaussian distribution of barrier heights and modified Richardson plot}

To explain the abnormalities observed in our Schottky diodes, we considered the barrier inhomogeneous, consisting of various patches of relatively lower or higher barriers with respect to a mean value.

Now, using the assumption above, the temperature dependence of barrier height can be attributed to inhomogeneity of barrier height. Since current transport across metal-semiconductor interface is a temperature-activated process, at low temperatures electrons are able to surmount the low barriers and therefore current transport will be dominated by current flowing through patches of lower SBH and a larger ideality factor. In other words, as the temperature increases more electrons have sufficient energy to overcome the higher barrier. Therefore the dominant barrier height will increase with the rise of temperature [2].

We modeled the barrier height inhomogeneities with a Gaussian distribution having a mean value $\overline{\phi_{b}}$ and standard deviation of $\delta_{s}$ which is expressed as

$$
P\left(\phi_{b}\right)=\frac{1}{\delta_{s} \sqrt{2 \pi}} \exp \left[-\frac{\left(\phi_{b}-\overline{\phi_{b}}\right)^{2}}{2 \delta_{s}^{2}}\right]
$$


The total current across a Schottky diode containing barrier inhomogeneities can be written as

$$
I(V)=\int_{-\infty}^{+\infty} I\left(\phi_{b}, V\right) P\left(\phi_{b}\right) d \phi_{b}
$$

where $I\left(\phi_{b}, V\right)$ is the current for a barrier of height $\phi_{b}$ at voltage $\mathrm{V}$ based on the ideal thermionic theory and $P\left(\phi_{b}\right)$ is the normalized distribution function giving the probability of the occurrence of barrier height $\phi_{b}$. By introducing $I\left(\phi_{b}, V\right)$ and $P\left(\phi_{b}\right)$ from Eqs. (1) and (7) in Eq. (8) the current of the Schottky diode with the modified barrier will be

$$
\begin{aligned}
I(V)= & A A^{*} T^{2} \exp \left[\frac{-q}{k T}\left(\overline{\phi_{b}}-\frac{q \delta_{s}^{2}}{2 k T}\right)\right] \\
& \times \exp \left(\frac{q V}{n_{a p} k T}\right)\left[1-\exp \left(\frac{-q V}{k T}\right)\right]
\end{aligned}
$$

Comparing Eqs. (1) and (9) leads to an expression for the apparent barrier height $\phi_{a p}$ as a function of the mean barrier and temperature.

$$
\phi_{a p}=\overline{\phi_{b}}-\frac{q \delta_{s}^{2}}{2 k T}
$$

According to Eq. (10), the plot $\phi_{a p}$ versus $q / 2 k T$ should be a straight line with the intercept determining $\overline{\phi_{b}}$ and the slope giving $\delta_{s}$. By using the barrier heights obtained from I-V measurements (Table I) the $\phi_{a p}$ versus $q / 2 k T$ plot is made and shown in Fig. 2 (a). It is obvious that the data fit nicely with a straight line and the intercept and slope of this straight line yields values for $\overline{\phi_{b}}$ and $\delta_{s}$ of $0.4548 \mathrm{eV}$ and 0.0607 in the $85-159 \mathrm{~K}$ range.

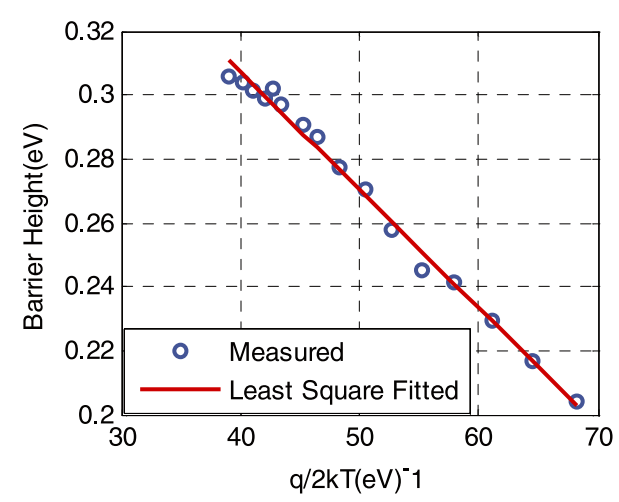

(a)

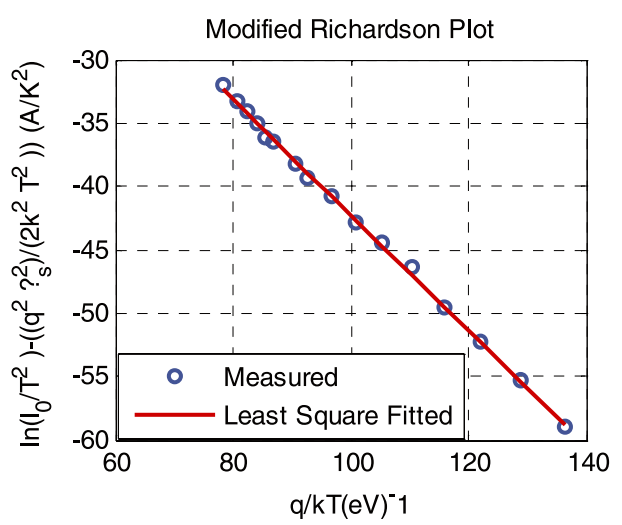

(b)

Fig. 2. (a) $\phi_{a p}$ versus $q / 2 k T$ curve and (b) Modified Richardson $\ln \left(I_{\circ} / T^{2}\right)-\left(q^{2} \delta_{s}^{2} / 2 k^{2} T^{2}\right)$ versus $q / k T$ plot for the PtSi/p-Si Schottky diode.

It was shown before in this paper that the conventional activation energy plot of the PtSi/p-Si diode deviates from linearity. To explain this discrepancy, Eq. (6) can be rewritten considering Eq. (10) as

$$
\ln \left(\frac{I_{\circ}}{T^{2}}\right)-\left(\frac{q^{2} \delta_{s}^{2}}{2 k^{2} T^{2}}\right)=\ln \left(A A^{*}\right)-\frac{q \overline{\phi_{b}}}{k T}
$$


and based on this equation the modified activation energy plot $\left(\ln \left(I_{\circ} / T^{2}\right)-\right.$ $\left(q^{2} \delta_{s}^{2} / 2 k^{2} T^{2}\right)$ versus $\left.q / k T\right)$ is made. This plot takes into account the variations of the barrier height and should give a straight line with the slope directly yielding the mean barrier $\left(\overline{\phi_{b}}\right)$ and the y-axis intercept $\ln \left(A A^{*}\right)$ giving Richardson constant $\left(A^{*}\right)$. Fig. $2(\mathrm{~b})$ shows the modified activation plot for $\mathrm{PtSi} / \mathrm{p}$-Si diode which is acceptably linear and from the slope and intercept of the fitted straight line $\overline{\phi_{b}}$ and $A^{*}$ were determined to be $0.4546 \mathrm{eV}$ and $31.47 \mathrm{Acm}^{-2} \mathrm{~K}^{-2}$ respectively. The value of $\overline{\phi_{b}}$ matches exactly with mean barrier height obtained from the $\phi_{a p}$ versus $q / 2 k T$ plot earlier and the value of Richardson constant is in great agreement with the theoretical value of $32 \mathrm{Acm}^{-2} \mathrm{~K}^{-2}$ for p-type Si.

\section{Conclusion}

In this paper the I-V characteristics of $\mathrm{PtSi} / \mathrm{p}-\mathrm{Si}$ Schottky diodes were measured in the temperature range of $85-136 \mathrm{~K}$. The electrical parameters of the diode such as ideality factor and barrier height were found to be strongly temperature-dependent. These departures from the classic thermionic emission theory can be well described by assuming the existence of barrier height inhomogeneities and modeling them with a Gaussian distribution. Using the $\phi_{a p}$ versus $q / 2 k T$ plot, the mean value and standard deviation of the Gaussian distribution of barrier heights were determined to be $0.4548 \mathrm{eV}$ and $0.0607 \mathrm{eV}$ respectively. The activation energy plot was seen to deviate from linearity but the modified Richardson plot which considers the Gaussian distribution of barrier heights can be fitted nicely with a straight line. The mean barrier height and the Richardson constant from the modified Richardson plot were determined to be $0.4546 \mathrm{eV}$ and $31.47 \mathrm{Acm}^{-2} \mathrm{~K}^{-2}$. The value of Richardson constant obtained from the modified Richardson plot is very close to the known value of $32 \mathrm{Acm}^{-2} \mathrm{~K}^{-2}$ for p-type Si. The linearity of the modified activation plot and the closeness of the calculated Richardson constant to its theoretical value are good reasons for the success of the Gaussian distribution model in explaining the temperature dependence of I-V characteristics of the $\mathrm{PtSi} / \mathrm{p}-\mathrm{Si}$ Schottky barrier diodes. 